\title{
Experimental Study on Autism Caused by Prenatal Exposure to Plasticizer DEHP
}

\author{
Boyang Hu, Yiting Song, Genan Liang, Yihuan Shao, Linling Qi, Xue Jiang, Yuemin Ding* \\ Department of Clinical Medicine, School of Medicine, Zhejiang University City College, Hangzhou, China
}

Email address:

dingyuemin@zucc.edu.cn (Yuemin Ding)

${ }^{*}$ Corresponding author

\section{To cite this article:}

Boyang Hu, Yiting Song, Genan Liang, Yihuan Shao, Linling Qi, Xue Jiang, Yuemin Ding. Experimental Study on Autism Caused by Prenatal Exposure to Plasticizer DEHP. Science Discovery. Vol. 7, No. 2, 2019, pp. 78-81. doi: 10.11648/j.sd.20190702.14

Received: March 5, 2019; Accepted: May 8, 2019; Published: May 23, 2019

\begin{abstract}
Objective: Epidemiological studies have found that exposure to plasticizers in the environment may be associated with autism in preschool children, but laboratory evidence is lacking. To determine whether prenatal exposure to DEHP, a plasticizer, causes autism in offspring mice. Methods: Pregnant mice were randomly divided into four groups: corn oil control group and low, medium and high concentration DEHP exposure group $(10 \mathrm{mg} / \mathrm{kg}, 50 \mathrm{mg} / \mathrm{kg}, 250 \mathrm{mg} / \mathrm{kg})$. Mice were received continuous intragastric administration of DEHP from the 3rd day to the 17th day of pregnancy. Behavioral characteristics of autism in offspring were assessed after birth. Results: The social ability of offspring was significantly decreased after prenatal exposure to moderate and high concentration DEHP. The female offspring showed repetitive stereotyped behavior and decreased spontaneous activities. Conclusion: Exposure to medium and high concentration of DEHP during embryonic stage can induce autism in offsprings.
\end{abstract}

Keywords: Di(2-ethylhexyl)phthalate, Autism Spectrum Disorder, Neurodevelopment

\section{胚胎期塑化剂DEHP暴露导致自闭症的实验研究}

\author{
胡博洋, 宋依婷, 梁戈楠, 邵艺欢, 戚林玲, 姜雪, 丁悦敏"
}

浙江大学城市学院医学院临床医学系, 杭州, 中国

\section{邮箱}

dingyuemin@zucc.edu.cn (丁悦敏)

摘要: 目的: 流行病学研究发现环境中塑化剂暴露可能与学龄前儿童自闭症的发病有关, 但缺乏实验室证据, 为明确 胚胎期塑化剂DEHP暴露是否导致小鼠自闭症的发生。方法: 孕鼠被随机分为4组: 玉米油对照组和低、中、高浓度DEHP 染毒组 $(10 \mathrm{mg} / \mathrm{kg}, 50 \mathrm{mg} / \mathrm{kg}, 250 \mathrm{mg} / \mathrm{kg})$, 自怀胎第3天起至第 17 天连续用DEHP进行灌胃染毒。仔鼠出生后, 用行为 学评估仔鼠自闭症行为学特性。结果: 胚胎期中高浓度DEHP染毒使仔鼠社交能力明显下降, 其中雌性仔鼠出现重复 刻板活动且自发活动明显减弱。结论：胚胎期中高浓度塑化剂DEHP暴露可致仔鼠自闭症的发生。

关键词: 邻苯二甲酸二乙基乙酯, 自闭症谱系障碍, 神经发育 


\section{1. 引言}

自闭症谱系障碍（autism spectrum disorder，ASD）是 一种神经发育障碍导致的疾病, 它的发病不仅与遗传因素 有关, 还与环境因素密不可分 [1-2]。动物神经行为发育最 为关键的时期是胚胎期, 此时环境中的毒素、药物等不良 因素极易影响胎儿的神经发育, 出生后发生自闭症的风险 显著升高[3]。流行病学研究发现, 环境中的一类塑化剂邻 苯二甲酸酯类化合物 (phthalic acid easters, PAEs) 可能与 学龄前儿童自闭症的发病有关 [4]。近年来, 进一步揭示ASD 的发病机制，探索PAEs与ASD之间的联系机制已成为该领 域热切关注的重要课题。邻苯二甲酸二乙基己基酯（Di (2-ethylhexyl) phthalate, DEHP) 是一种典型的PAEs, 王永 俊等研究发现, 哺乳期暴露于 $50 、 500 \mathrm{mg} / \mathrm{kg}$ DEHP的雄性 子代大鼠海马内突触可塑性蛋白PSD95、CREB、PKA、 CAMKII的mRNA表达水平下降，提示DEHP可影响子代大 鼠的神经系统发育, 进而对空间学习能力造成损害[5]。徐 晓虹等研究发现, 母鼠从妊娠第七天至断乳前 (产后21天) 每天灌胃染毒DEHP10、50、200mg/kg 可抑制青春期仔鼠的 活动性, 使其产生焦虑, 该焦虑状态可以延续至成年后[6]。 此外, DEHP暴露还可损伤青春期雄鼠的空间学习记忆能力 [7]。但胚胎期DEHP暴露是否导致仔鼠自闭症的产生尚不 清楚。为解答该问题, 本课题拟在胚胎期DEHP暴露的小鼠 模型上察看仔鼠自闭症相关神经行为特点, 探讨胚胎期 DEHP暴露这一环境因素是否为自闭症发生的诱因。

\section{2. 材料与方法}

\section{1. 实验动物}

从浙江医学科学院实验动物中心购得清洁级健康ICR 小鼠 50 只, 含 40 只雌鼠和 10 只雄鼠, 体重为 $25-30 \mathrm{~g}$ 。

\section{2. 主要仪器及试剂}

DEHP（美国sigma），玉米油（中国金龙鱼），动物 行为学分析软件及系统（荷兰Noldus）。

\section{3. 实验分组及模型制备}

将40只雌鼠随机且分批与雄鼠合笼, 合笼之后记录怀 胎雌鼠的个数和怀胎时间。将孕鼠随机分为 4 组, 分别是 玉米油对照组和低、中、高浓度DEHP染毒组 ( $10 \mathrm{mg} / \mathrm{kg}$, $50 \mathrm{mg} / \mathrm{kg}, 250 \mathrm{mg} / \mathrm{kg}$ ), 染毒组采取灌胃方式进行染毒, 每天一次, 建立DEHP胚胎期暴露模型, 而玉米油组孕鼠 则灌胃等量玉米油以对照。灌胃自怀胎第 3 天起至第 17 天, 共15天。

\section{4. 行为学观察}

\subsection{1. 三箱社交实验}

第一阶段: 将被测仔鼠放入三个箱子相互连通的实验 箱中任意探索10分钟。第二阶段: 在右侧箱体的鼠笼中放 入一只同性陌生鼠 1 号, 让被测仔鼠任意探索 10 分钟。第 三阶段: 在左侧箱体的鼠笼中再放入一只同性陌生鼠 2 号,
让被测仔鼠任意探索10分钟。记录三个阶段里仔鼠在三个 箱子区域中逗留的时间。

\subsection{2. 埋弹珠实验}

第一阶段: 在鼠笼中铺上 $10 \mathrm{~cm}$ 厚的玉米芯垫料, 让仔 鼠在鼠笼中适应 10 分钟。第二阶段: 在玉米芯垫料上放上 15 个玻璃珠, 分五排, 每排 3 个。将仔鼠放入笼中 20 分钟 后统计其所埋弹珠的数目。

\subsubsection{Y迷宫实验}

在Y迷宫中心放入一只 5 周龄的小鼠让其自由探索8分 钟, 用摄像观察并记录仔鼠进入迷宫臂的顺序, 如果仔鼠 相邻三次分别进入不同的迷宫臂则记录为正确的一组, 正 确的组数除以总组数则为仔鼠y迷宫实验的正确率, 完成 一只仔鼠的测试后需使用 $75 \%$ 酒精擦拭迷宫消除气味。

\section{5. 数据统计}

所有数据均用平均值 \pm 标准误差 $(\overline{\mathrm{X}} \pm \mathrm{S})$ 表示。用 SPSS20.0软件进行统计分析, 对比两组数据用单因素方差 分析法, 多组用登尼特法, $P<0.05$ 表示在统计学上有显 著性意义。

\section{3. 结果}

\subsection{DEHP染毒影响仔鼠社会交互能力}

三箱社交实验第二阶段结果显示, 当右侧箱体的鼠笼 中放入同性陌生鼠 1 号之后, 玉米油对照组仔鼠无论雌雄 在该侧的停留时间均显著长于左侧空白箱体 $(P<0.05)$, 这种行为特征反映了正常小鼠的社会交互能力。而中高浓 度DEHP $(50 \mathrm{mg} / \mathrm{ml}$ 和 $250 \mathrm{mg} / \mathrm{ml})$ 染毒组仔鼠在小鼠所在 的右侧箱体内的停留时间的较玉米油对照组显著缩短, 表 现为左右两侧箱体停留时间无差异（图1A，1B）, 说明 DEHP染毒对仔鼠的社会交互能力产生了负面影响。

第三阶段结果显示, 当左侧箱体的鼠笼中放入一只新 的同性陌生鼠 2 号之后，玉米油对照组仔鼠无论雌雄在该 侧的停留时间均显著长于右侧箱体 $(P<0.05)$ 。而中高 浓度DEHP $(50 \mathrm{mg} / \mathrm{ml}$ 和 $250 \mathrm{mg} / \mathrm{ml})$ 染毒组仔鼠在2号鼠所 在左侧箱体内的停留时间的较玉米油对照组显著缩短, 表 现为左右两侧箱体停留时间无差异（图2A，2B）。以上 两个阶段实验结果说明中高浓度DEHP可以导致仔鼠社会 交互能力下降。

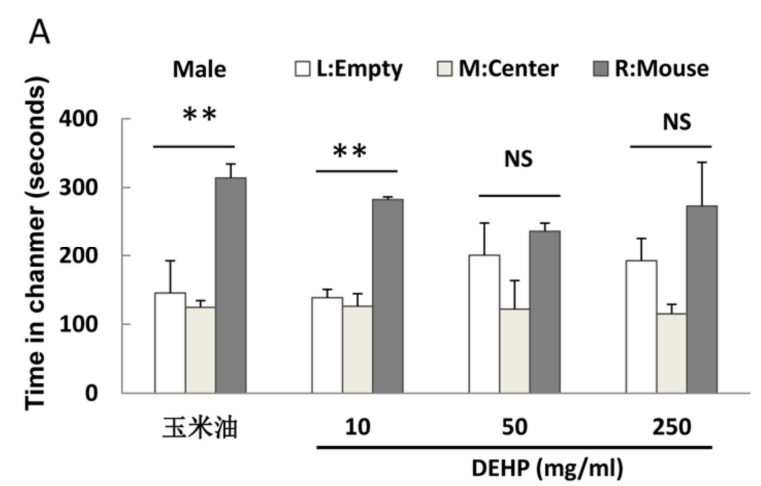


B

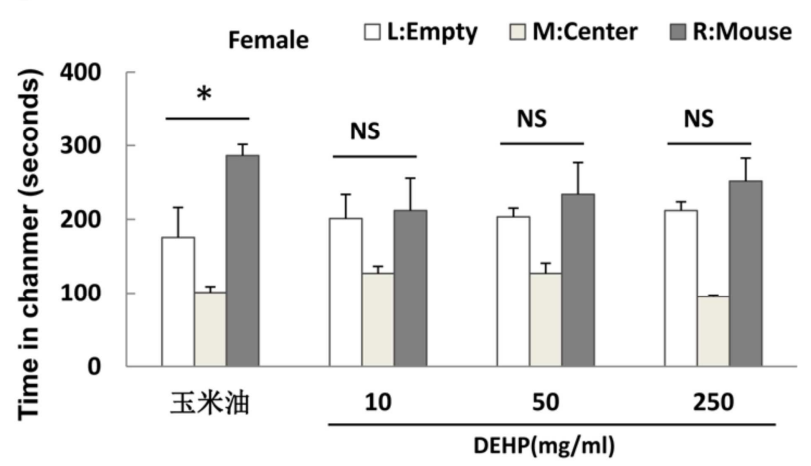

图1 DEHP染毒对仔鼠三箱社交实验第二阶段结果的影响。

（A）实验第二阶段雄性仔鼠在不同箱体内停留时间的统计图; （B） 第二阶段雌性仔鼠在不同箱体内停留时间的统计图。与对侧箱体停留时 间组相比, $* P<0.05, * * P<0.01, \quad(\mathrm{n}=8-14)$
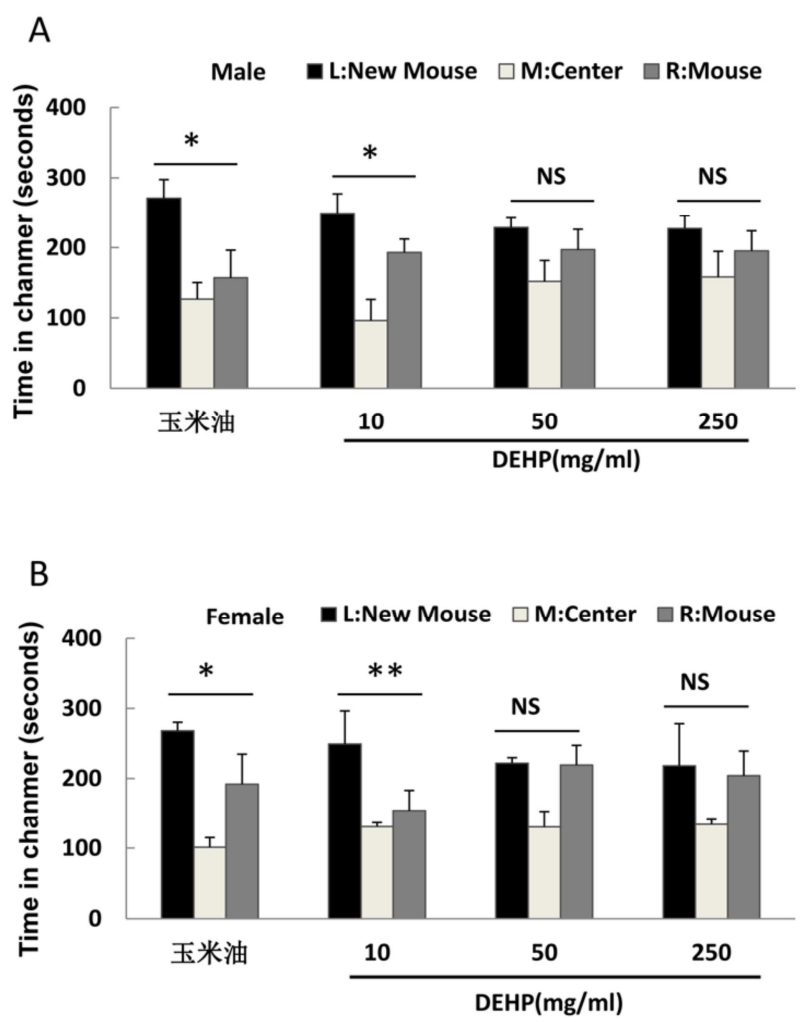

图2 DEHP染毒对仔鼠三箱社交实验第三阶段结果的影响。

（A）实验第三阶段雄性仔鼠在不同箱体内停留时间的统计图; (B) 第三阶段雌性仔鼠在不同箱体内停留时间的统计图。与对侧箱体停留时 间组相比, $* P<0.05, * * P<0.01, \quad(\mathrm{n}=8-14)$

\subsection{DEHP染毒使雌性仔鼠产生重复刻板行为}

除了社交能力下降, 自闭症动物还会出现典型的重复 刻板行为。将小鼠放置于有深色弹珠的笼子里, 自闭症小 鼠倾向于掩埋更多的弹珠。我们的实验结果发现, DEHP $(50 \mathrm{mg} / \mathrm{ml})$ 染毒的䧳性仔鼠埋弹珠百分率高于同性别玉 米油对照组 $(P<0.05)$, 但雄性仔鼠组间未见较大差别 （图3A，3B）。说明DEHP染毒使雌性仔鼠产生重复刻板 行为。
A

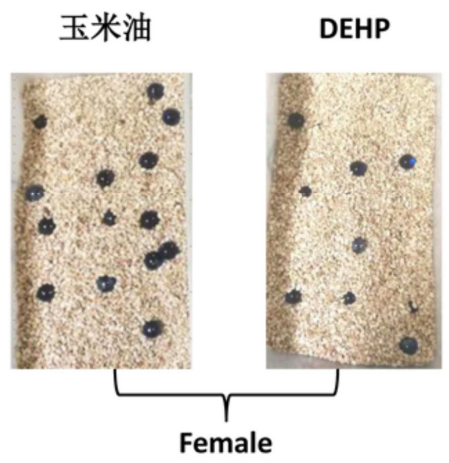

B

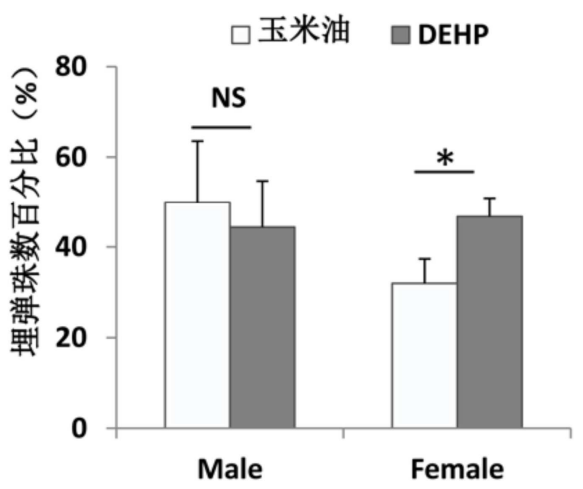

图3 DEHP染毒对仔鼠重复刻板行为的影响。

（A） DEHP染毒后仔鼠埋弹珠实验照片; （B）埋弹珠实验统计图。 与玉米油组相比, $* \mathrm{P}<0.05, * * \mathrm{P}<0.01, \quad(\mathrm{n}=8-14)$

\subsection{DEHP染毒影响仔鼠自发活动能力}

$\mathrm{Y}$ 迷宫通过检测动物的自发活动频率反映实验动物对 新异空间的探索能力, 自闭症动物的自发活动频率会相对 减少。本实验结果显示DEHP $(50 \mathrm{mg} / \mathrm{ml})$ 染毒的雌性仔鼠 自发交替率较玉米油组显著减少 $(P<0.01)$, 同样, 该 差异却未能在雄性仔鼠中出现（图4）。该结果说明DEHP 染毒使雌性仔鼠的自发活动和对新异空间的探索能力下 降。

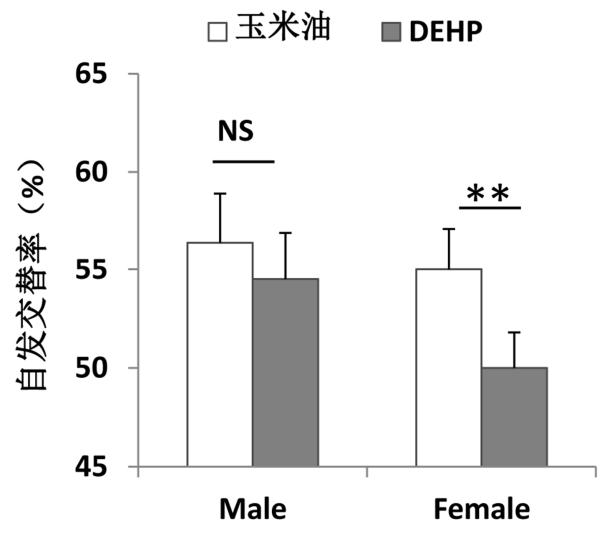

图4 DEHP染毒对仔鼠自发活动能力的影响。 


\section{4. 讨论}

邻苯二甲酸酯（PAEs）是常见的一种塑化剂, 被广 泛用于食品、医疗、化妆品等生产过程。PAEs通过氢键 或范德华力联接塑料中的聚合物, 非常容易逸出并进入人 类生存环境中。DEHP是PAEs中的一种, 研究发现DEHP 可通过多种途径进入人体, 对神经系统、生殖系统、呼吸 系统都具有潜在毒性[8]。2012年, Testa等发现48例自闭 症谱系障碍 (ASD) 儿童的尿液中DEHP的代谢产物明显 比正常对照儿童高, 首次提出了DEHP与ASD之间的联系 [4]。截至2015年12月共有 5 项独立的流行病学研究探讨了 该问题，其中3项研究结果支持Testa等的结论[9]。已有的 研究发现孕期母体摄入的DEHP可通过胎盘和乳汁进入子 代体内, 影响子代的神经行为发育[6], 导致空间学习记忆 能力下降 [5,10-11]等。但在动物模型上直接研究胚胎 DEHP暴露与子代自闭症的关系尚未见报道。

胚胎期人和哺乳类动物的血脑屏障尚未发育完全, 脂 溶性药物往往比于水溶性药物更容易进入脑组织中, 从而 对神经系统的发育造成影响[12]。因此, 脂溶性较高的 DEHP能较容易地通过血脑屏障。在妊娠期中, 对于胎盘 屏障而言, 胎盘对药物的转运并无屏障作用, 其对药物的 通透性与一般的毛细血管无明显差别, 几乎所有的药物都 可以穿透胎盘进入胎儿体内, 从而发生药理学变化导致胎 儿各个系统发生变化[13]。器官形成期是胚胎神经系统发 育的关键期, 因此, 我们选择建立DEHP胚胎期暴露模型 进行实验。DEHP对神经系统发育造成了一系列影响。有 研究发现DEHP可以干扰神经元和神经胶质细胞的增殖分 化过程, 受孕第 8 天暴露于DEHP的大鼠胚胎神经管发育畸 形, 亦会诱导青年大鼠的多动症[14]。流行病学研究发现, 患有注意缺陷多动症的儿童尿液中存在DEHP的代谢产物 MEHP[15]。

本项目实验结果显示胚胎期中高浓度DEHP染毒对子 代小鼠的社交能力产生损害, 使得雌性仔鼠出现自闭症特 有的重复刻板行为及自发性活动下降、对新异环境失去探 索本能等神经行为改变, 与流行病学研究结果相符。

\section{5. 结论}

综上所述, 本项目首次在大鼠模型上探讨了胚胎期塑 化剂DEHP暴露与仔鼠自闭症发病之间的关系, 发现胚胎 期中高浓度DEHP染毒可导致子代雌雄小鼠的社交能力均 受损。此外, 雌性小鼠出现重复刻板行为及自发性活动下 降的改变, 但该变化在雄性小鼠中未被观察到, 其详细机 制仍需进一步深入研究。

\section{致谢}

本文为国家级大学生创新创业训练计划《胚胎期塑化 剂DEHP暴露导致儿童自闭症的实验研究》(201813021013) 的阶段性成果之一。

\section{参考文献}

[1] 仇子龙. 自闭症的神经生物学研究 科学, 2015,67(6):20-23.

[2] Tchaconas A, Adesman A. Autistic Spectrum Disorder. Current Opinion in Pediatrics, 2013,25(1):130-144.

[3] Nuttall J R. The plausibility of maternal toxicant exposure and nutritional status as contributing factors to the risk of autism spectrum disorders. Nutr Neurosci, 2017,20(4):209-218.

[4] Testa C, Nuti F, Hayek J, De Felice C, Chelli M, Rovero P, Latini G, Papini A M. Di-(2-ethylhexyl) phthalate and autism spectrum disorders. ASN Neuro, 2012,4(4):223-229.

[5] 王永俊, 李永宁, 马宁, 贾旭东. DEHP暴露对子代大鼠空 间学习记忆能力和海马突触可塑性相关蛋白基因表达的影 响. 中国食品卫生杂志. 2015,27(3):237-241.

[6] 徐晓虹, 竹庆杰, 杨艳玲, 阮琴. 出生前后DEHP暴露对小 鼠神经行为的影响. 浙江师范大学学报 (自然科学 版）.2015,38(1):15-22.

[7] 徐晓虹, 沈超, 周易, 秦泽旭, 梅成龙, 施剑. 增塑剂 DEHP的发育和神经毒性研究进展. 浙江师范大学学报 (自 然科学版） 2013, 2:209-212.

[8] Bang du Y, Lee I K, Lee B M. Toxicological characterization of phthalic Acid. Toxicol Res, 2011,27(4):191-203.

[9] Jeddi M Z, Janani L, Memari A H, Akhondzadeh S, Yunesian M. The role of phthalate esters in autism development: A systematic review. Environ Res, 2016,151:493-504.

[10] Lin H, Yuan K, Li L, Liu S, Li S, Hu G, Lian Q Q, Ge R S. In Utero Exposure to Diethylhexyl Phthalate Affects Rat Brain Development: A Behavioral and Genomic Approach. Int J Environ Res Public Health, 2015,12(11):13696-13710.

[11] Li X J, Jiang L, Chen L, Chen H S, Li X. Neurotoxicity of dibutyl phthalate in brain development following perinatal exposure: a study in rats. Environ Toxicol Pharmacol, 2013,36(2):392-402.

[12] Abbott N J. Blood-brain barrier structure and function and the challenges for CNS drug delivery. J Inherit Metab Dis, 2013,36(3):437-449.

[13] Kalisch-Smith J I, Simmons D G, Pantaleon M, Moritz K M. Sex differences in rat placental development: from pre-implantation to late gestation. Biol Sex Differ, 2017,8:17.

[14] Masuo Y, Morita M, Oka S, Ishido M. Motor hyperactivity caused by a deficit in dopaminergic neurons and the effects of endocrine disruptors: a study inspired by the physiological roles of PACAP in the brain. Regul Pept, 2004,123(1-3):225-234.

[15] Engel S M, Miodovnik A, Canfield R L, Zhu C, Silva M J, Calafat A M, Wolff M S. Prenatal phthalate exposure is associated with childhood behavior and executive functioning. Environ Health Perspect, 2010,118(4):565-571. 